\title{
Straining flow of a micellar surfactant solution
}

\author{
C. J. W. Breward and P. D. Howell \\ Mathematical Institute, University of Oxford, Oxford. OX1 3LB. UK
}

(Received 5 April 2004)

\begin{abstract}
We present a mathematical model describing the distribution of monomer and micellar surfactant in a steady straining flow beneath a fixed free surface. The model includes adsorption of monomer surfactant at the surface and a single-step reaction whereby $n$ monomer molecules combine to form each micelle. The equations are analysed asymptotically and numerically and the results are compared with experiments.

Previous studies of such systems have often assumed equilibrium between the monomer and micellar phases, i.e. that the reaction rate is effectively infinite. Our analysis shows that such an approach inevitably fails under certain physical conditions and also cannot accurately match some experimental results. Our theory provides an improved fit with experiments and allows the reaction rates to be estimated.
\end{abstract}

\section{Introduction}

A surfactant is an amphiphilic chemical. When dissolved in a bulk liquid phase (usually water), it is energetically favourable for surfactant molecules to arrange themselves with their hydrophobic components expelled through any liquid-air interface present. This adsorption of surfactant at a free surface reduces the local surface tension. Nonuniform adsorption thus results in a surface tension gradient that drives a liquid flow and, hence, affects the transport of surfactant molecules. Consequently, when surfactant solution flows beneath a free surface, the fluid dynamics and surface chemistry are, in general, intimately coupled.

If enough molecules are present locally, they can also reduce their free energy by combining in aggregates called micelles, in which their hydrophobic parts are shielded from the surrounding liquid. The minimum concentration required for the formation of micelles is called the Critical Micelle Concentration (CMC). Micelles may take several forms including spheres and sheets [23]; see figure 1. Although different-sized micelles may occur, one size is usually far more stable than the others [23].

In this paper we consider the flow of a micellar surfactant solution (i.e. with bulk concentration above the CMC) beneath a free surface. If the free surface expands, the surface concentration of surfactant decreases and hence must be replenished from the bulk. This reduces the local bulk concentration and, potentially, causes micelles in a neighbourhood of the interface to dissociate. The process of interface adsorption from micellar solutions under dynamic conditions is described further in [21].

The coupling between surface expansion and micellar breakdown has been investigated 

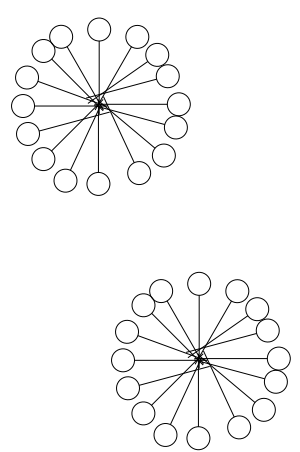
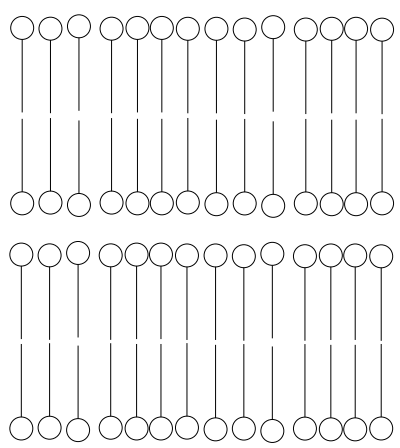

FIGURE 1. Schematic showing cross sections through spherical and sheet micelles.

using an experimental apparatus, known as the OverFlowing Cylinder (OFC), in which water is pumped up a vertical cylinder and flows gently over the rim. The resulting stagnation-point flow is easily accessible for non-invasive measurements of surface velocity, surface tension and surface concentration of surfactant [3, 19, 20]. Introduction of even a small amount of surfactant to the system induces a large increase in the surface expansion rate. This can be explained as follows. Expansion of the surface causes depletion of surfactant near to the rim of the cylinder as compared to the centre. The resulting surface tension gradient is responsible for accelerating the free surface. The theory behind this process for surfactant solution below the CMC is given in [6] and [15].

Conventional wisdom suggests that, above the CMC, the air/liquid interface is loaded with surfactant and thus rendered immobile (indeed, it is common to apply no-slip boundary conditions at surfactant-loaded interfaces when modelling soap-films; see [22] for example). Alternatively one might argue that, with surfactant readily available in the form of micelles, any nonuniformity in surface concentration should be rapidly replenished, so that surface tension gradients are effectively eliminated. The OFC investigations show that neither of these hypotheses is correct: the large surfactant-induced surface expansion persists even when the bulk concentration is much higher than the CMC. In figure 2, for example, we see that the maximum surface expansion rate $\theta$ for the surfactant $\mathrm{C}_{14} \mathrm{TAB}$ is achieved above the CMC. This suggests that the overflowing cylinder operates in a regime where the local surfactant concentration beneath the free surface is sub-CMC even when the bulk concentration is not.

In this paper we derive a simple mathematical model describing the flow of micellar surfactant solution beneath a gas-liquid interface. The model incorporates surfactant in three different forms: individual monomer molecules in solution, micelles in the bulk, and molecules adsorbed at the interface. Our aim is to show how surface expansion causes micelles to break down. Indeed, our theory allows the timescale for micelle breakdown to be inferred from the results of OFC experiments.

Our main simplifying assumption is to allow only micelles of a single fixed size to form via the combination of $n$ individual monomers. There are many more complicated models in the literature, describing aggregates whose sizes vary as they gain or lose monomer molecules $[21,13,4,9]$. All these models neglect fluid motion and thus surfactant transport by convection. Our model is similar to that used in [10] and amounts to lumping all 


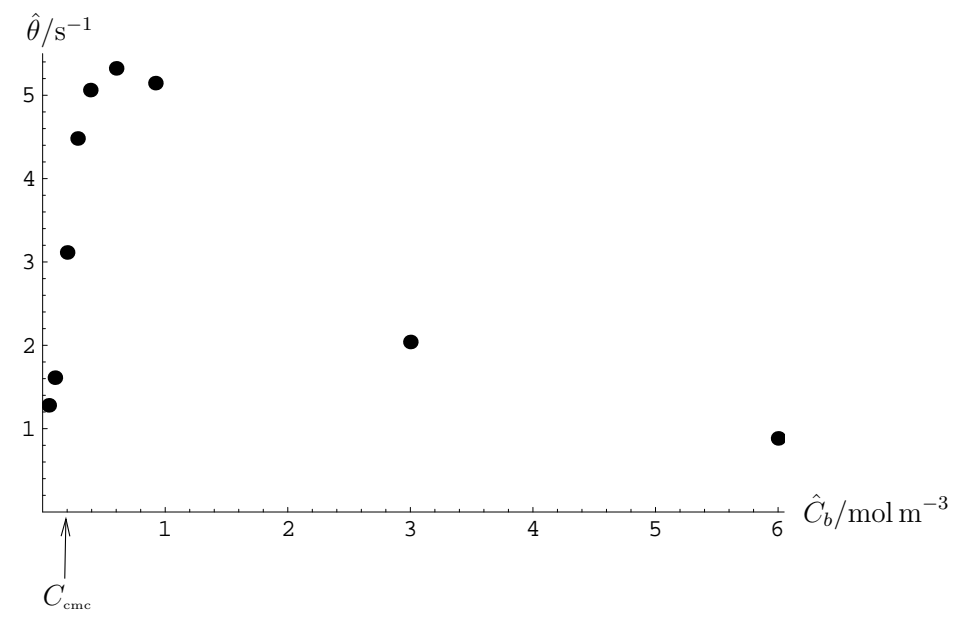

Figure 2. Surface expansion rate $\theta$ at the centre of the OFC versus bulk concentration $C_{b}$ of surfactant $\left(\mathrm{C}_{14} \mathrm{TAB}+0.1 \mathrm{M} \mathrm{NaBr}\right)$. The $\mathrm{CMC}$ for this surfactant solution is $0.2 \mathrm{~mol} \mathrm{~m}^{-3}$. The data are reported in [24] and reproduced by kind permission of the authors.

such intermediate stages into a single effective reaction between monomers and micelles. It has the advantage of limiting to two the number of rate constants to be fitted from experiments. It is also simple enough to be applied to situations where the monomer and micelle concentrations are far from their equilibrium values.

In section 2, we derive our general governing equations and boundary conditions in dimensionless form. Typical behaviour of the solutions to these equations is illustrated in section 3 by considering a particularly simple limit in which they can be solved analytically. In general, although exact analytical solutions are not available, the equations may be simplified considerably by taking relevant asymptotic limits. This is done in section 4 , where the simplified models are validated via comparison with numerical solutions of the full model. Further validation is provided in section 5 by comparing our asymptotic and numerical solutions with experimental data. Finally, in section 6 , we discuss our results and draw our conclusions.

\section{Mathematical model}

\subsection{Governing equations}

We suppose that each micelle is an aggregation of $n$ monomer molecules, where $n$ is typically in the range $80-100$ for spherical micelles [2]. We model this process as a single chemical reaction of the form

$$
\begin{aligned}
& k_{1} \\
& n \mathrm{MON} \rightleftharpoons \mathrm{MIC}, \\
& k_{-1}
\end{aligned}
$$

neglecting all intermediate reaction steps. The law of mass action implies that the rate, $j$, at which surfactant changes phase from monomer to micelle is

$$
j=k_{1} \hat{C}^{n}-k_{-1} \hat{C}_{m},
$$


where the concentrations of monomer and micellar surfactant are denoted by $\hat{C}$ and $\hat{C}_{m}$ respectively. Here and throughout, ${ }^{\wedge}$ is used to indicate dimensional variables. Both phases are advected with the fluid velocity $\hat{\boldsymbol{u}}$ while diffusing, with diffusion coefficients denoted by $D$ and $D_{m}$ respectively. Hence the two concentrations satisfy the reactionadvection-diffusion equations

$$
\begin{gathered}
\frac{\partial \hat{C}}{\partial \hat{t}}+\hat{\boldsymbol{u}} \cdot \hat{\nabla} \hat{C}=D \hat{\nabla}^{2} \hat{C}-n j, \\
\frac{\partial \hat{C}_{m}}{\partial \hat{t}}+\hat{\boldsymbol{u}} \cdot \hat{\nabla} \hat{C}_{m}=D_{m} \hat{\nabla}^{2} \hat{C}_{m}+j,
\end{gathered}
$$

where the fluid is assumed to be incompressible so that $\hat{\nabla} \cdot \hat{\boldsymbol{u}}=0$.

In this paper we restrict our attention to radially-symmetric flow beneath a flat interface. We therefore use cylindrical polar coordinates $(\hat{r}, \hat{z})$ with the free surface at $\hat{z}=0$ and the $\hat{z}$-axis pointing vertically downward into the liquid. Far away from the interface, the two concentrations are assumed to be in equilibrium, with the net concentration of surfactant molecules equal to a known constant $\hat{C}_{b}$. The corresponding values of $\hat{C}_{m}$ and $\hat{C}$ are given by

$$
\hat{C}_{m} \sim \frac{k_{1}}{k_{-1}} \hat{C}^{n}, \quad \hat{C}+n \frac{k_{1}}{k_{-1}} \hat{C}^{n} \rightarrow \hat{C}_{b}, \quad \text { as } \quad \hat{z} \rightarrow \infty .
$$

The surface concentration of surfactant molecules adsorbed at the interface is denoted by $\hat{\Gamma}$. If surface diffusion is neglected (see [6] for the justification), then $\hat{\Gamma}$ evolves through advection along the interface, with surface velocity $\hat{\boldsymbol{u}}_{s}(\hat{r})=\hat{\boldsymbol{u}}(\hat{r}, 0)$, and replenishment by monomer from the bulk:

$$
D \frac{\partial \hat{C}}{\partial \hat{z}}=\frac{\partial \hat{\Gamma}}{\partial \hat{t}}+\hat{\nabla} \cdot\left(\hat{\boldsymbol{u}}_{s} \hat{\Gamma}\right) \quad \text { at } \quad \hat{z}=0
$$

Micelles, whose outer surfaces are hydrophilic, are not directly adsorbed without first breaking up into monomers, so the flux of $\hat{C}_{m}$ at the interface must be zero:

$$
\frac{\partial \hat{C}_{m}}{\partial \hat{z}}=0 \quad \text { at } \quad \hat{z}=0 .
$$

Finally, we assume that the timescale for adsorption of monomer onto the free surface is small (i.e. the monomer and adsorbed phases are in thermodynamic equilibrium) so there is a functional relationship between $\hat{\Gamma}$ and $\hat{C}$ at the interface. We use the Langmuir isotherm [1]

$$
\hat{\Gamma}=\frac{\Gamma_{\text {sat }} \hat{C}}{k+\hat{C}} \quad \text { at } \quad \hat{z}=0,
$$

where $\Gamma_{\text {sat }}$ and $k$ are material properties of the surfactant.

The initial profiles of both concentrations are also supposed to be given:

$$
\hat{C}=\hat{C}_{I}(\hat{z}), \quad \hat{C}_{m}=\hat{C}_{m I}(\hat{z}) \quad \text { at } \quad \hat{t}=0 .
$$

In general the fluid velocity $\hat{\boldsymbol{u}}$ must be found by solving the Navier-Stokes equations and appropriate free-surface conditions, where the surface tension is related to surface concentration of surfactant. To simplify matters, though, we suppose for the moment that 
$\hat{\boldsymbol{u}}$ is a given function of $\hat{r}, \hat{z}$ and $\hat{t}$. Then (2.3)-(2.9) form a closed system of equations and boundary conditions for $\hat{C}, \hat{C}_{m}$ and $\hat{\Gamma}$.

\subsection{Nondimensionalisation}

From (2.5), we deduce the following expressions for the equilibrium concentrations far from the interface:

$$
\frac{n \hat{C}_{m}}{C_{\mathrm{cmc}}}=\left(\frac{\hat{C}}{C_{\mathrm{cmc}}}\right)^{n}, \quad\left(\frac{\hat{C}}{C_{\mathrm{cmc}}}\right)+\left(\frac{\hat{C}}{C_{\mathrm{cmc}}}\right)^{n}=\frac{\hat{C}_{b}}{C_{\mathrm{cmc}}}
$$

where

$$
C_{\mathrm{cmc}}=\left(\frac{k_{-1}}{n k_{1}}\right)^{1 /(n-1)} .
$$

If $\hat{C}_{b}<C_{\text {cmc }}$ then, since $n$ is a large integer (of order 100), (2.10) implies that $\hat{C} \sim \hat{C}_{b}$ and $\hat{C}_{m} \sim 0$. On the other hand, if $\hat{C}_{b}>C_{\text {cmc }}$ then we deduce that $\hat{C} \sim C_{\text {cmc }}$ and $\hat{C}_{m} \sim\left(\hat{C}_{b}-C_{\text {cmc }}\right) / n$. We can therefore identify $C_{\text {cmc }}$ with the critical micelle concentration above which micelles form.

We use this insight in nondimensionalising the problem as follows:

$$
\hat{\boldsymbol{u}}=\frac{D}{L} \boldsymbol{u}, \quad(\hat{r}, \hat{z})=L(r, z), \quad \hat{t}=\frac{L^{2}}{D} t, \quad \hat{C}=C_{\mathrm{cmc}} C, \quad \hat{C}_{m}=\frac{C_{\mathrm{cmc}}}{n} C_{m}, \quad \hat{\Gamma}=\Gamma_{\mathrm{sat}} \Gamma
$$

where the length-scale $L$ can be determined later, depending on the geometry under consideration. The resulting dimensionless equations and boundary conditions read

$$
\begin{gathered}
\frac{\partial C}{\partial t}+\boldsymbol{u} \cdot \nabla C=\nabla^{2} C-K\left(C^{n}-C_{m}\right), \\
\frac{\partial C_{m}}{\partial t}+\boldsymbol{u} \cdot \nabla C_{m}=\mathcal{D} \nabla^{2} C_{m}+K\left(C^{n}-C_{m}\right),
\end{gathered}
$$

with

$$
\begin{gathered}
C_{m} \sim C^{n}, \quad C+C^{n} \rightarrow C_{b} \quad \text { as } z \rightarrow \infty, \\
\frac{1}{S} \frac{\partial C}{\partial z}=\frac{\partial \Gamma}{\partial t}+\nabla \cdot\left(\boldsymbol{u}_{s} \Gamma\right), \quad \frac{\partial C_{m}}{\partial z}=0, \quad \Gamma=\frac{C}{\beta+C} \quad \text { at } \quad z=0, \\
C=C_{0}(z), \quad C_{m}=C_{m 0}(z) \quad \text { at } \quad t=0 .
\end{gathered}
$$

Along with $n$, there are five dimensionless groups, defined by

$$
\mathcal{D}=\frac{D_{m}}{D}, \quad K=\frac{L^{2} k_{-1}}{D}, \quad C_{b}=\frac{\hat{C}_{b}}{C_{\mathrm{cmc}}}, \quad S=\frac{\Gamma_{\mathrm{sat}}}{L C_{\mathrm{cmc}}}, \quad \beta=\frac{k}{C_{\mathrm{cmc}}} .
$$

Depending on the flow and geometry under consideration, it may be possible to reduce these to four by making a suitable choice of $L$.

\subsection{Application to stagnation point flow}

Now we apply this model (2.13-2.17) to the overflowing cylinder experiment. The cylinder used has radius $4 \mathrm{~cm}$ and, within $2 \mathrm{~cm}$ of the axis of the cylinder, the experimentally- 
measured leading-order flow is that of a stagnation point (see [6]), with velocity field

$$
\hat{\boldsymbol{u}}=\frac{\theta \hat{r}}{2} \boldsymbol{e}_{r}-\theta \hat{z} \boldsymbol{e}_{z}
$$

where $\boldsymbol{e}_{r}$ and $\boldsymbol{e}_{z}$ are unit vectors in the $\hat{r}$ - and $\hat{z}$-directions respectively. To determine $\theta$ theoretically, it is necessary to solve for the flow all the way out to the rim of the cylinder [15]. Instead, as in [6], we suppose that $\theta$ is known (from experimental measurements) and try to determine the surfactant concentrations.

For this flow field, the appropriate nondimensionalisation is $\hat{\boldsymbol{u}}=\theta L \boldsymbol{u}$, which can be reconciled with (2.12) by choosing the lengthscale $L$ appropriately:

$$
L=\sqrt{D / \theta}
$$

If the concentrations $C$ and $C_{m}$ are assumed only to depend on $z$, the dimensionless model reads

$$
\begin{gathered}
\frac{\mathrm{d}^{2} C}{\mathrm{~d} z^{2}}+z \frac{\mathrm{d} C}{\mathrm{~d} z}=K\left(C^{n}-C_{m}\right), \\
\mathcal{D} \frac{\mathrm{d}^{2} C_{m}}{\mathrm{~d} z^{2}}+z \frac{\mathrm{d} C_{m}}{\mathrm{~d} z}=-K\left(C^{n}-C_{m}\right),
\end{gathered}
$$

with

$$
\begin{gathered}
\frac{\mathrm{d} C}{\mathrm{~d} z}=S \frac{C}{\beta+C} \quad \text { at } \quad z=0, \\
\frac{\mathrm{d} C_{m}}{\mathrm{~d} z}=0 \quad \text { at } z=0, \\
C_{m} \sim C^{n}, \quad C+C^{n} \rightarrow C_{b} \text { as } z \rightarrow \infty .
\end{gathered}
$$

The dimensionless parameters $n, \beta$ and $\mathcal{D}$ depend only on the surfactant under consideration. Of these, $n$ is large and $\beta$ is usually small, say $O(0.01)$. The ratio of the diffusion coefficients may be estimated from the ratio of the molecular weights, which suggests $\mathcal{D} \sim n^{-1 / 3} \approx 0.2$. The remaining parameters vary from experiment to experiment, depending on the bulk concentration and flow rate, but $S$ and $C_{b}$ are both typically order one. The final parameter $K$ is proportional to the reaction rate $k_{-1}$ which is unknown. If micelle breakdown is assumed to occur very rapidly, then we can take the limit $K \rightarrow \infty$, in which case the monomer and micelle phases are everywhere in equilibrium; see [24]. In this paper, we are particularly interested in the non-equilibrium regime, where $K$ is finite and the timescale for micelle breakdown is an important limiting process.

\section{Paradigm problem}

We first consider a particularly straightforward, although physically unrealistic, limit in which equations (2.20) and (2.21) can be integrated analytically. From this we gain valuable insight into the relevant asymptotic scalings that can be used to simplify the problem in general.

Suppose the diffusion coefficients of the two phases are equal, so $\mathcal{D}=1$. Suppose further that the dimensionless reaction rate $K$ is very large. Then we deduce from (2.20) and (2.21) that the two phases are everywhere in equilibrium, i.e.

$$
C_{m} \equiv C^{n} \text {. }
$$


Then, by adding (2.20) and (2.21), we obtain a linear differential equation for $C+C^{n}$, whose solution is

$$
C+C^{n}=C_{b}-\left(C_{b}-C_{0}-C_{0}^{n}\right) \operatorname{erfc}(z / \sqrt{2}),
$$

where $C_{0}$ is shorthand for the subsurface monomer concentration $C(0)$. This remaining unknown is fixed by applying the boundary condition (2.22), which leads to

$$
C_{b}=C_{0}+C_{0}^{n}+S \sqrt{\frac{\pi}{2}}\left(\frac{C_{0}+n C_{0}^{n}}{\beta+C_{0}}\right) .
$$

It is readily shown that, given $S$ and $\beta,(3.3)$ defines a one-to-one relationship between $C_{0}$ and $C_{b}$. Once $C_{0}$ has been found, the concentration $C$ is given by the algebraic equation (3.2).

First suppose $C_{0}<1$, so that $C_{0}^{n} \ll 1$. Then (3.3) reduces to a quadratic equation for $C_{0}$, whose solution is

$$
C_{0} \sim \frac{1}{2}\left\{C_{b}-\beta-S \sqrt{\frac{\pi}{2}}+\sqrt{\left(C_{b}-\beta-S \sqrt{\pi / 2}\right)^{2}+4 \beta C_{b}}\right\} .
$$

The hypothesis $C_{0}<1$ is hence only valid when the bulk concentration is bounded by

$$
C_{b}<1+\frac{S}{1+\beta} \sqrt{\frac{\pi}{2}}
$$

Next we analyse equation (3.2) in this limit. If $C_{b}<1$, then the system is sub-micellar $(C<1)$ everywhere, so the solution of $(3.2)$ is approximately

$$
C \sim C_{b}-\left(C_{b}-C_{0}\right) \operatorname{erfc}(z / \sqrt{2}), \quad C_{m} \sim 0 .
$$

If $C_{b}>1$, then (3.6) is only valid in $z<Z$, where $Z$ satisfies $C(Z)=1$, that is

$$
\operatorname{erfc}(Z / \sqrt{2})=\frac{C_{b}-1}{C_{b}-C_{0}} .
$$

In $z>Z$, the balance in (3.2) switches to

$$
C \sim 1, \quad C_{m} \sim C_{b}-1-\left(C_{b}-C_{0}\right) \operatorname{erfc}(z / \sqrt{2}) .
$$

For higher values of $C_{b}$, not satisfying (3.5), the hypothesis $C_{0}^{n} \approx 0$ fails, with the result that (3.4) over-predicts $C_{0}$. Instead, the leading-order approximate solution of (3.3) becomes

$$
C_{0} \sim 1-\frac{1}{n}\left\{\log \left(\frac{n S}{1+\beta} \sqrt{\frac{\pi}{2}}\right)+\log \left(C_{b}-1-\frac{S}{1+\beta} \sqrt{\frac{\pi}{2}}\right)\right\} .
$$

In this limit, the solution is everywhere micellar, so the approximation (3.8) applies right up to the free surface $z=0$.

Figure 3 shows comparisons between the asymptotic approximations (3.6)-(3.9) and numerical solutions of (3.2) and (3.3). With the parameter values used, the critical value of $C_{b}$ given by (3.5) is 2.24. In general, the liquid is partitioned into two regions, separated by the free boundary $z=Z$, which satisfies (3.7). The value of $Z$ for the parameters used in figure $3(\mathrm{a})$ is 0.82 . In $z<Z$, there are effectively no micelles and the monomer 

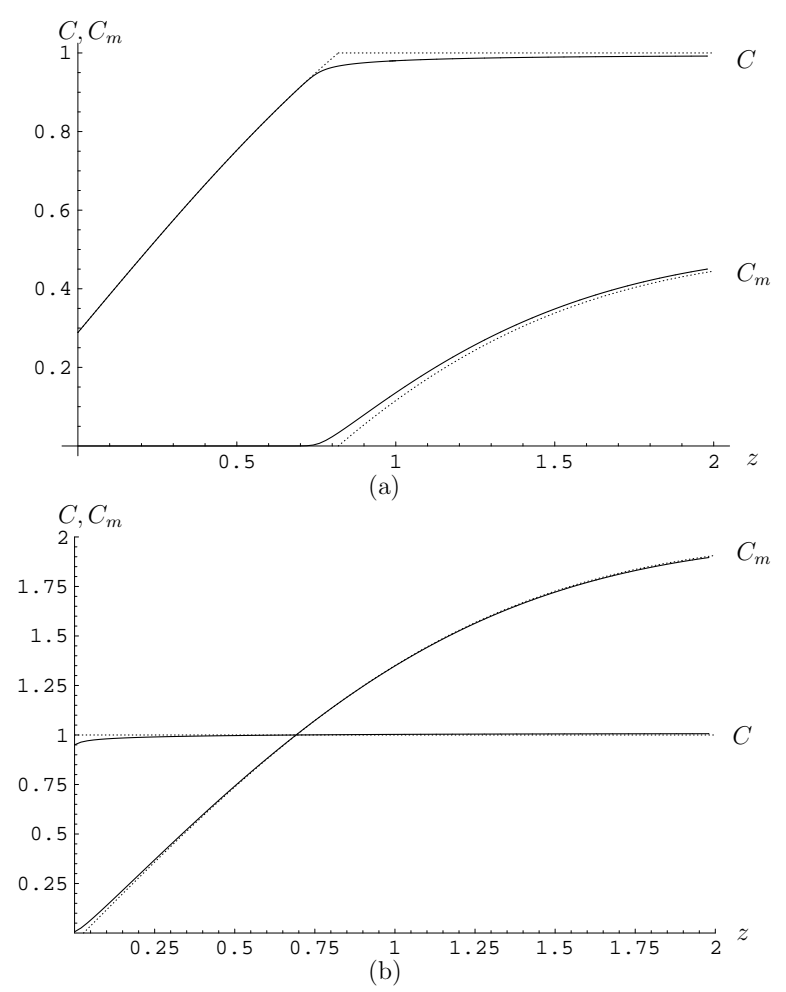

Figure 3. Monomer and micelle concentrations, $C$ and $C_{m}$, versus depth $z$ with bulk concentration (a) $C_{b}=1.5$, (b) $C_{b}=3$. The solid line is the exact solution (3.2)-(3.3) with $n=100$; the dotted line is the large- $n$ asymptotic solution (3.6)-(3.8). The other parameter values are $\beta=0.01$ and $S=1$.

concentration is below the CMC while, in $z>Z$, the micelle concentration is positive and the monomer concentration is approximately at the CMC. At bulk concentrations below the CMC, the latter region ceases to exist and the solution is sub-micellar everywhere, that is $Z \rightarrow \infty$. At the other extreme (see figure $3(\mathrm{~b})$ ), if the bulk concentration exceeds the limit given in (3.5), then $Z$ is effectively zero, the monomer concentration is constant and micelles exist all the way up to $z=0$.

There is a further complication: we have as yet made no attempt to impose the micelle flux boundary condition (2.23). This condition is approximately satisfied if the subsurface concentration is sub-micellar, so that (3.6) applies near $z=0$. It is no longer satisfied, though, if $C_{0}$ becomes sufficiently close to 1 . Specifically,

$$
\frac{\partial C_{m}}{\partial z}(0) \sim n C_{0}^{n-1} \frac{\partial C}{\partial z}(0) \sim \frac{n S C_{0}^{n}}{\beta+C_{0}},
$$

which is order one if

$$
C_{0} \sim 1-\frac{1}{n} \log \left(\frac{n S}{1+\beta}\right) \Rightarrow C_{b} \sim 1+\sqrt{\frac{\pi}{2}}\left(\frac{S}{1+\beta}+1\right) .
$$

At bulk concentration higher than this, the limit $K \rightarrow \infty$ necessarily fails in a neighbourhood of $z=0$. It is clear in figure $3(\mathrm{~b})$, for example, that $\partial C_{m} / \partial z$ is nonzero at 
$z=0$. This illustrates the danger of assuming in advance that the monomer and micelle phases are everywhere in equilibrium.

\section{Asymptotic analysis}

\subsection{The limit $n \rightarrow \infty$}

Now we return to the full equations (2.20)-(2.24) and use the insight gained from the simplified model in section 3 to construct approximate solutions in asymptotic limits relevant to real-life surfactants.

In all surfactant systems of interest, the number $n$ of monomers contained in each micelle is a large parameter. Hence we can, as in section 3 , partition the fluid into a sub-micellar region where $C<1$, and a micellar region where $C \approx 1$, divided by the free boundary $z=Z$. In the former, $C^{n}$ is exponentially small, so the reduced equations

$$
z<Z, \quad C<1, \quad K C_{m}=\mathcal{D} \frac{\mathrm{d}^{2} C_{m}}{\mathrm{~d} z^{2}}+z \frac{\mathrm{d} C_{m}}{\mathrm{~d} z}, \quad K C_{m}=-\frac{\mathrm{d}^{2} C}{\mathrm{~d} z^{2}}-z \frac{\mathrm{d} C}{\mathrm{~d} z}
$$

are valid to all algebraic orders in $1 / n$. To analyse the case where $C$ is close to 1 , we set $C=1+\phi / n$, so

$$
C^{n} \sim \mathrm{e}^{\phi}\left\{1-\frac{\phi^{2}}{2 n}+\frac{\phi^{3}(8+3 \phi)}{24 n^{2}}+\cdots\right\} \quad \text { as } \quad n \rightarrow \infty .
$$

Hence the version of (4.1) that holds in the micellar region is

$$
\begin{gathered}
z>Z, \quad C=1+\frac{\phi}{n}, \\
K\left\{C_{m}-\mathrm{e}^{\phi}\left(1-\frac{\phi^{2}}{2 n}+\cdots\right)\right\}=\mathcal{D} \frac{\mathrm{d}^{2} C_{m}}{\mathrm{~d} z^{2}}+z \frac{\mathrm{d} C_{m}}{\mathrm{~d} z}=-\frac{1}{n}\left(\frac{\mathrm{d}^{2} \phi}{\mathrm{d} z^{2}}+z \frac{\mathrm{d} \phi}{\mathrm{d} z}\right) .
\end{gathered}
$$

Our analysis in the remainder of this section is based on (4.1) and (4.2)

\subsection{The limit $K \rightarrow \infty$}

Before attempting to find and match solutions of (4.1) and (4.2), we first consider the simplified equations that result from the equilibrium limit $K \rightarrow \infty$. In this limit, $C_{m}=$ $C^{n}$ everywhere and we find, as in section 3 , that the micelle concentration in $z<Z$ is effectively zero, so (4.1) becomes

$$
z<Z, \quad C_{m}=0, \quad \frac{\mathrm{d}^{2} C}{\mathrm{~d} z^{2}}+z \frac{\mathrm{d} C}{\mathrm{~d} z}=0 .
$$

The solution, satisfying $C(Z)=1$, is

$$
C=C_{0}+\frac{\left(1-C_{0}\right) \operatorname{erf}(z / \sqrt{2})}{\operatorname{erf}(Z / \sqrt{2})},
$$

where $C_{0}=C(0)$ as before. An equation for $C_{0}$ comes from the boundary condition (2.22):

$$
S \sqrt{\frac{\pi}{2}} \operatorname{erf}(Z / \sqrt{2})=\frac{\left(1-C_{0}\right)\left(\beta+C_{0}\right)}{C_{0}} .
$$


The leading-order equations in $z>Z$ are

$$
z>Z, \quad C=1, \quad \mathcal{D} \frac{\mathrm{d}^{2} C_{m}}{\mathrm{~d} z^{2}}+z \frac{\mathrm{d} C_{m}}{\mathrm{~d} z}=0,
$$

to be solved with the conditions

$$
C_{m}(Z)=0, \quad C_{m} \rightarrow C_{b}-1 \quad \text { as } \quad z \rightarrow \infty .
$$

We assume here that $C_{b}>1$, so that $Z<\infty$. Then the solution of (4.6), (4.7) is

$$
C_{m}=\left(C_{b}-1\right)\left\{1-\frac{\operatorname{erfc}(z / \sqrt{2 \mathcal{D}})}{\operatorname{erfc}(Z / \sqrt{2 \mathcal{D}})}\right\}
$$

It remains to determine the location of the free boundary $z=Z$. In principle this could be done by asymptotic matching of the two solutions (4.4) and (4.8) through a boundary layer near $z=Z$; see section 4.3 for details. Here we can circumvent this step by ensuring that surfactant is conserved across $z=Z$. Since $C \sim 1$, the flux of surfactant crossing $z=Z$ from above is purely in the form of micelles. In $z<Z$, though, there are no micelles, so surfactant enters through $z=Z$ only in monomer form. By setting these fluxes equal to each other,

$$
\frac{\mathrm{d} C}{\mathrm{~d} z}=\mathcal{D} \frac{\mathrm{d} C_{m}}{\mathrm{~d} z} \quad \text { at } \quad z=0
$$

we obtain the following transcendental equation for $Z$ :

$$
\frac{\operatorname{erf}(Z / \sqrt{2}) \mathrm{e}^{-(1-\mathcal{D}) Z^{2} / 2 \mathcal{D}}}{\operatorname{erfc}(Z / \sqrt{2 \mathcal{D}})}=\frac{1-C_{0}}{\left(C_{b}-1\right) \sqrt{\mathcal{D}}}
$$

where $C_{0}$ satisfies (4.5). Notice that (4.9) agrees with (3.7) if $\mathcal{D}=1$.

In figure 4, we compare the approximate solutions (4.4) and (4.8) with numerical solutions of the full equations (2.20)-(2.24) with $n$ and $K$ large but finite. As $K$ and $n$ increase, $C_{0}$ increases and the solutions tend to the infinite $K$ and $n$ limit. The second graph indicates the peril of working in the infinite $K$ and $n$ limit: for moderate values micelles exist right up to the free surface. The asymptotic solution (4.4), (4.5), (4.8) and (4.9) is the nondimensional version of that presented in [24].

\subsection{The limit $K=O(1)$}

Now we investigate the effects of the micellar and monomer phases being out of equilibrium. In $z<Z$, we first solve (4.1) for $C_{m}(z)$, with $C_{m}^{\prime}(0)=0$ :

$$
C_{m}=B_{1} \mathrm{e}^{-z^{2} / 2 \mathcal{D}} \Phi\left(\frac{1+K}{2}, \frac{1}{2}, \frac{z^{2}}{2 \mathcal{D}}\right),
$$

where $\Phi$ is a confluent hypergeometric function [14, page 1084], and $B_{1}$ is an integration constant. Next we solve (4.1) for $C(z)$ :

$$
C=C_{0}+B_{2} \sqrt{\frac{\pi}{2}} \operatorname{erf}\left(\frac{z}{\sqrt{2}}\right)-K \sqrt{\frac{\pi}{2}} \int_{0}^{z}\left\{\operatorname{erf}\left(\frac{z}{\sqrt{2}}\right)-\operatorname{erf}\left(\frac{\zeta}{\sqrt{2}}\right)\right\} C_{m}(\zeta) \mathrm{e}^{\zeta^{2} / 2} \mathrm{~d} \zeta,
$$




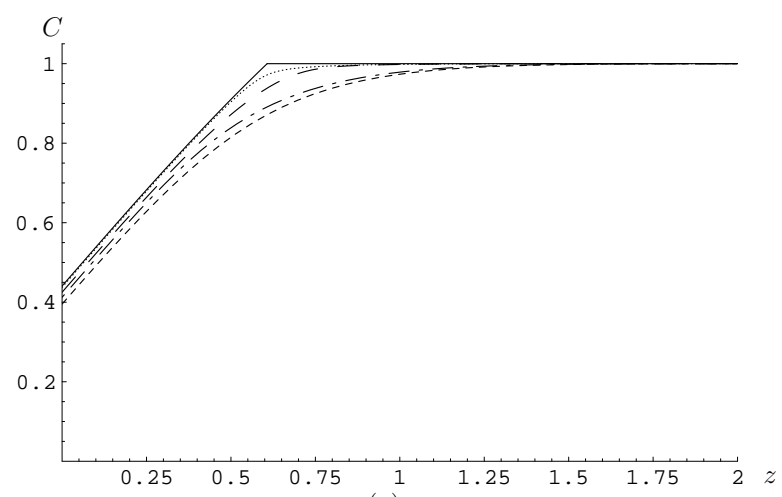

(a)

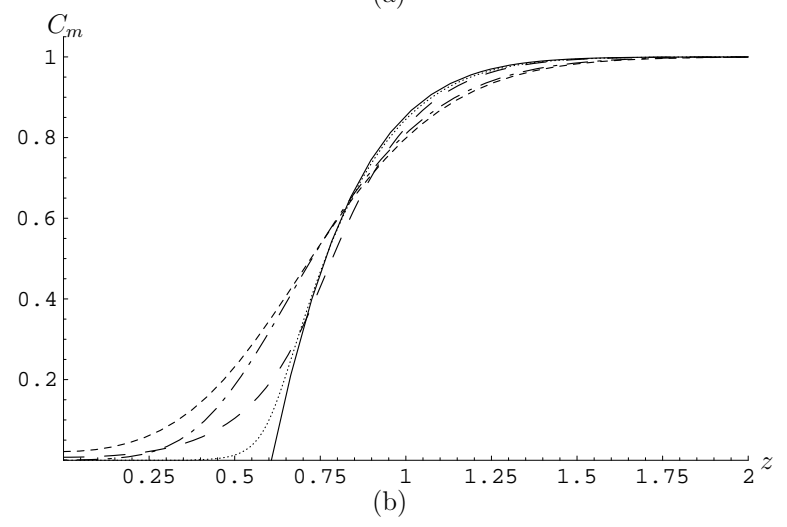

Figure 4. Concentration of (a) monomer and (b) micelles versus depth $z$ for $n=10, K=10$ (small-dashed line), $n=100, K=10$ (large-dashed line), $n=10, K=100$ (dot-dashed line) and $n=100, K=100$ (dotted line). The solid line shows the asymptotic solution for infinite $n$ and $K$. The other parameter values are $S=1, \beta=0.01$ and $C_{b}=2$.

where the integration constants are related through the boundary condition (2.22) on $z=0$ :

$$
C_{0}=\frac{\beta B_{2}}{S-B_{2}} \text {. }
$$

In $z>Z$, we solve (4.2) for $C_{m}$ and $\phi$ as asymptotic expansions of the form

$$
C_{m} \sim C_{m 0}+n^{-1} C_{m 1}+\cdots, \quad \phi \sim \phi_{0}+n^{-1} \phi_{1}+\cdots .
$$

The leading-order solutions are

$$
\begin{aligned}
C_{m 0} & =C_{b}-1+\left(\mathrm{e}^{B_{3}}-C_{b}+1\right) \frac{\operatorname{erfc}(z / \sqrt{2 \mathcal{D}})}{\operatorname{erfc}(Z / \sqrt{2 \mathcal{D}})}, \\
\phi_{0} & =\log \left(C_{m 0}\right),
\end{aligned}
$$

where the integration constant $B_{3}$ is defined such that

$$
\phi_{0}(Z)=B_{3}, \quad C_{m 0}(Z)=\mathrm{e}^{B_{3}}, \quad C_{m 0}^{\prime}(Z)=-\sqrt{\frac{2}{\pi \mathcal{D}}} \frac{\left(\mathrm{e}^{B_{3}}-C_{b}+1\right) \mathrm{e}^{-Z^{2} / 2 \mathcal{D}}}{\operatorname{erfc}(Z / \sqrt{2 \mathcal{D}})} .
$$


Now, to determine the unknown constants $C_{0}, B_{3}$ and $Z$, we match the solutions (4.10), (4.11), (4.13) and (4.14) across an inner layer near $z=Z$. To explore this layer we perform the rescaling

$$
z=Z+y / \sqrt{n}, \quad \phi(z)=\psi(y), \quad C_{m}(z)=g(y),
$$

so that $\psi$ and $g$ satisfy the equations

$$
K\left\{g-\mathrm{e}^{\psi}\left(1-\frac{\psi^{2}}{2 n}+\cdots\right)\right\}=\mathcal{D} n \frac{\mathrm{d}^{2} g}{\mathrm{~d} y^{2}}+(Z \sqrt{n}+y) \frac{\mathrm{d} g}{\mathrm{~d} y}=-\frac{\mathrm{d}^{2} \psi}{\mathrm{d} y^{2}}-\left(\frac{Z}{\sqrt{n}}+\frac{y}{n}\right) \frac{\mathrm{d} \psi}{\mathrm{d} y}
$$

We seek the solutions as asymptotic expansions of the form

$$
g \sim g_{0}+n^{-1 / 2} g_{1}+n^{-1} g_{2}+\cdots, \quad \psi \sim \psi_{0}+n^{-1 / 2} \psi_{1}+n^{-1} \psi_{2}+\cdots .
$$

At leading order, we find that $g_{0}^{\prime \prime}(y)=0$ and hence, by matching as $y \rightarrow \infty$,

$$
g_{0}(y)=\mathrm{e}^{B_{3}} .
$$

Then $\psi_{0}$ satisfies the differential equation

$$
\frac{\mathrm{d}^{2} \psi_{0}}{\mathrm{~d} y^{2}}=K\left(\mathrm{e}^{\psi_{0}}-\mathrm{e}^{B_{3}}\right)
$$

with $\psi_{0} \rightarrow B_{3}$ as $y \rightarrow \infty$. By choosing the arbitrary translation in $y$ appropriately, we may write the solution in the form

$$
\sqrt{2 K} y=\int_{0}^{\psi_{0}} \frac{\mathrm{d} s}{\sqrt{\mathrm{e}^{s}-\mathrm{e}^{B_{3}}+\mathrm{e}^{B_{3}}\left(B_{3}-s\right)}}
$$

For matching purposes, we need the behaviour of $\psi_{0}$ as $y \rightarrow-\infty$, which we obtain from

$$
\sqrt{2 K} \mathrm{e}^{B_{3} / 2} y \sim 2 \sqrt{B_{3}-1-\psi_{0}}+I\left(B_{3}\right) \text { as } \quad \psi_{0} \rightarrow-\infty,
$$

where the function $I(B)$ is defined by

$$
I(B)=\int_{1-B}^{\infty}\left(\frac{1}{\sqrt{s+B-1}}-\frac{1}{\sqrt{s+B-1+\mathrm{e}^{-s-B}}}\right) \mathrm{d} s-\int_{0}^{1-B} \frac{\mathrm{d} s}{\sqrt{s+B-1+\mathrm{e}^{-s-b}}} .
$$

Hence we find

$$
\psi_{0} \sim-\frac{1}{2} K \mathrm{e}^{B_{3}} y^{2}+\sqrt{\frac{K}{2}} \mathrm{e}^{B_{3} / 2} I\left(B_{3}\right) y+B_{3}-1-\frac{I\left(B_{3}\right)^{2}}{4}+\cdots \quad \text { as } \quad y \rightarrow-\infty .
$$

Proceeding to $O\left(n^{-1 / 2}\right)$, we find that $g_{1}^{\prime \prime}(y)=0$ and hence, by matching as $y \rightarrow \infty$,

$$
g_{1}=-y \sqrt{\frac{2}{\pi \mathcal{D}}} \frac{\left(\mathrm{e}^{B_{3}}-C_{b}+1\right) \mathrm{e}^{-Z^{2} / 2 \mathcal{D}}}{\operatorname{erfc}(Z / \sqrt{2 \mathcal{D}})}+B_{4}
$$

where $B_{4}$ is another integration constant.

Now we are finally in a position to match the solution in this inner layer with that in $z<Z$. First, the trivial equations for $g_{0}$ and $g_{1}$ imply that $C_{m}$ and $C_{m}^{\prime}$ are both 
continuous across $z=Z$ to leading order:

$$
C_{m}(Z) \sim \mathrm{e}^{B_{3}}, \quad C_{m}^{\prime}(Z) \sim-\sqrt{\frac{2}{\pi \mathcal{D}}} \frac{\left(\mathrm{e}^{B_{3}}-C_{b}+1\right) \mathrm{e}^{-Z^{2} / 2 \mathcal{D}}}{\operatorname{erfc}(Z / \sqrt{2 \mathcal{D}})} .
$$

Next, we expand our three-term inner solution for $C \sim 1+n^{-1} \psi_{0}$ in outer variables:

$$
C \sim 1-\frac{1}{2} K \mathrm{e}^{B_{3}}(z-Z)^{2}+\sqrt{\frac{K}{2 n}} \mathrm{e}^{B_{3} / 2} I\left(B_{3}\right)(z-Z)+O\left(n^{-1}\right) .
$$

The leading-order boundary conditions for $C$ are, therefore

$$
C(Z)=1, \quad C^{\prime}(Z)=0 .
$$

For relatively large values of $K$, it might be useful to include the $O\left(n^{-1 / 2}\right)$ correction to $C^{\prime}(Z)$ implied by (4.25). To avoid awkward calculations involving the unwieldy function $I(B)$, we do not bother to do so in this paper.

Now, applying the conditions (4.26) to (4.11), we obtain

$$
\begin{gathered}
B_{2}=K \int_{0}^{Z} \mathrm{e}^{z^{2} / 2} C_{m}(z) \mathrm{d} z \\
1-C_{0}=K \sqrt{\frac{\pi}{2}} \int_{0}^{Z} \operatorname{erf}\left(\frac{z}{\sqrt{2}}\right) C_{m}(z) \mathrm{e}^{z^{2} / 2} \mathrm{~d} z .
\end{gathered}
$$

Then substitution for $C_{m}$ from (4.10) leads to two equations for $B_{1}$ and $B_{2}$ :

$$
\begin{gathered}
B_{2}=K B_{1} \int_{0}^{Z} \mathrm{e}^{(\mathcal{D}-1) z^{2} / 2 \mathcal{D}} \Phi\left(\frac{1+K}{2}, \frac{1}{2}, \frac{z^{2}}{2 \mathcal{D}}\right) \mathrm{d} z \\
K B_{1} \sqrt{\frac{\pi}{2}} \int_{0}^{Z} \operatorname{erf}\left(\frac{z}{\sqrt{2}}\right) \mathrm{e}^{(\mathcal{D}-1) z^{2} / 2 \mathcal{D}} \Phi\left(\frac{1+K}{2}, \frac{1}{2}, \frac{z^{2}}{2 \mathcal{D}}\right) \mathrm{d} z=1-C_{0} .
\end{gathered}
$$

A third equation is obtained by eliminating $B_{3}$ from (4.24):

$$
\begin{gathered}
\sqrt{\frac{\pi \mathcal{D}}{2}} \operatorname{erfc}\left(\frac{Z}{\sqrt{2 \mathcal{D}}}\right) \mathrm{e}^{Z^{2} / 2 \mathcal{D}} C_{m}^{\prime}(Z)=C_{b}-1-C_{m}(Z) \\
\Rightarrow \quad B_{1} \sqrt{\frac{\pi}{2 \mathcal{D}}} Z \operatorname{erfc}\left(\frac{Z}{\sqrt{2 \mathcal{D}}}\right)\left\{(1+K) \Phi\left(\frac{3+K}{2}, \frac{3}{2}, \frac{Z^{2}}{2 \mathcal{D}}\right)-\Phi\left(\frac{1+K}{2}, \frac{1}{2}, \frac{Z^{2}}{2 \mathcal{D}}\right)\right\} \\
=C_{b}-1-B_{1} \mathrm{e}^{-Z^{2} / 2 \mathcal{D}} \Phi\left(\frac{1+K}{2}, \frac{1}{2}, \frac{Z^{2}}{2 \mathcal{D}}\right) .
\end{gathered}
$$

Now, given $\mathcal{D}, \beta, S, C_{b}$ and $K$, we have to solve the four coupled equations (4.12), (4.29), (4.30) and (4.31) for $B_{1}, B_{2}, C_{0}$ and $Z$. As before, a single transcendental equation for $Z$ may be obtained by eliminating all the other unknowns, but it is too lengthy to be worth reproducing here.

In figure 5, we compare the approximate solutions for large $n$ and finite $K$ with the approximate solutions for large $n$ and large $K$ and the numerical solution for finite $n$ and $K$. With the parameter values chosen, micelles exist right up to the free surface, but show a marked increase near $z=Z$. The large $n$ and finite $K$ asymptotics captures well the behaviour of $C$ and $C_{m}$ at all depths. The peril of assuming equilibrium between 


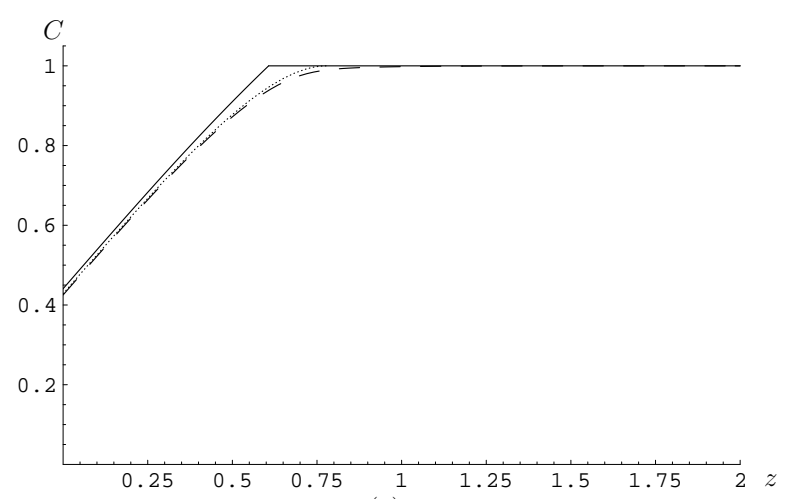

(a)

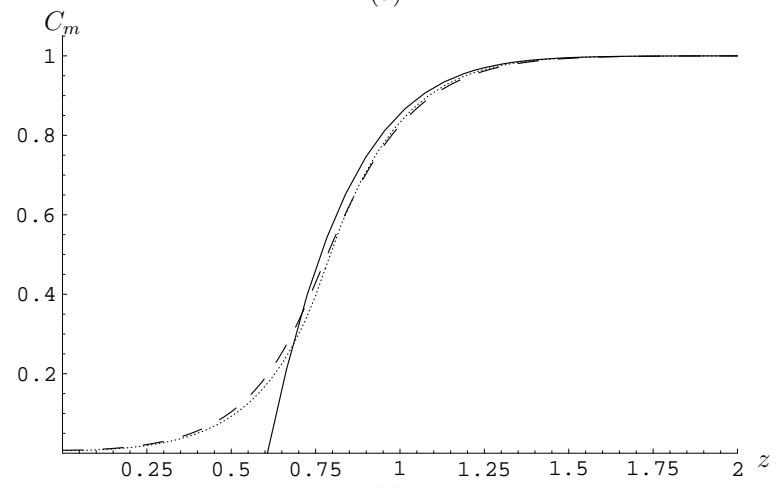

(b)

Figure 5. (a) Monomer and (b) micellar surfactant concentrations versus depth $z$. The dashed line shows the numerical solution for $K=10, n=100$, the dotted line shows the asymptotic solution for large $n$ and $K$ finite, and the solid line shows the solution for infinite $n$ and $K$. The other parameter values are $\mathcal{D}=0.2, S=1, \beta=0.01$ and $C_{b}=2$.

monomer and micelles is again apparent, since the infinite $n$ and $K$ asymptotic solution has no micelles closer to the free surface than $z=Z$.

\subsection{Summary}

In this section we have presented several asymptotic limits of the model (2.20)-(2.24). By comparing the solutions to the asymptotic problems with numerical solutions to (2.20)(2.24), we showed that the infinite- $n$ asymptotic solution gives a good approximation to the model both for $K$ infinite (compare the dotted and solid lines in figure 4) and for $K$ finite (see figure 5 ). Since the infinite- $n$ solution captures well the behaviour of the actual solution to $(2.20)-(2.24)$, in the next section we use the models presented in $\S 4.2$ and $\S 4.3$ to test the theory against experimental results.

In a real surfactant solution, $\beta$ is typically small, and this may be used to simplify further the transcendental equations derived above. It transpires, though, that three different dominant balances may prevail as $\beta \rightarrow 0$, depending on the size of $Z$ (which must itself be found as part of the solution). The slight simplification in the equations is 


\begin{tabular}{cccc}
\hline \hline Parameter & Units & Value for $\mathrm{C}_{14} \mathrm{TAB}$ & Value for $\mathrm{C}_{16} \mathrm{TAB}$ \\
\hline$C_{\mathrm{cmc}}$ & $\mathrm{mol} \mathrm{m}^{-3}$ & 0.2 & 0.035 \\
$\Gamma_{\text {sat }}$ & $\mathrm{mol} \mathrm{m}^{-2}$ & $3.6 \times 10^{-6}$ & $4.0 \times 10^{-6}$ \\
$k$ & $\mathrm{~mol} \mathrm{~m}^{-3}$ & 0.004 & 0.00028 \\
$D$ & $\mathrm{~m}^{2} \mathrm{~s}^{-1}$ & $4.5 \times 10^{-10}$ & $5 \times 10^{-10}$ \\
$D_{m}$ & $\mathrm{~m}^{2} \mathrm{~s}^{-1}$ & $9 \times 10^{-11}$ & $10^{-10}$ \\
\hline$n$ & - & 100 & 100 \\
$\beta$ & - & 0.02 & 0.008 \\
$\mathcal{D}$ & - & 0.2 & 0.2 \\
\hline \hline
\end{tabular}

Table 1. Approximate physical parameter values for $\mathrm{C}_{14} \mathrm{TAB}$ and for $\mathrm{C}_{16} \mathrm{TAB}$.

$\mathrm{C}_{14} \mathrm{TAB}$

\begin{tabular}{cc}
$\hat{C}_{b}\left(\mathrm{~mol} \mathrm{~m}^{-3}\right)$ & $\hat{\Gamma}\left(\mathrm{mol} \mathrm{m}^{-2}\right)$ \\
\hline 0.047 & $0.70 \times 10^{-6}$ \\
0.06 & $0.93 \times 10^{-6}$ \\
0.077 & $1.10 \times 10^{-6}$ \\
0.084 & $1.19 \times 10^{-6}$ \\
0.10 & $1.26 \times 10^{-6}$ \\
0.11 & $1.36 \times 10^{-6}$ \\
0.14 & $1.54 \times 10^{-6}$ \\
0.177 & $1.70 \times 10^{-6}$ \\
0.244 & $1.95 \times 10^{-6}$ \\
0.312 & $2.17 \times 10^{-6}$ \\
0.40 & $2.44 \times 10^{-6}$ \\
0.511 & $2.75 \times 10^{-6}$ \\
0.55 & $2.90 \times 10^{-6}$ \\
0.71 & $3.19 \times 10^{-6}$ \\
0.91 & $3.39 \times 10^{-6}$ \\
1.165 & $3.58 \times 10^{-6}$
\end{tabular}

$\mathrm{C}_{16} \mathrm{TAB}$

\begin{tabular}{cc}
\hline$\hat{C}_{b}\left(\mathrm{~mol} \mathrm{~m}^{-3}\right)$ & $\hat{\Gamma}\left(\mathrm{mol} \mathrm{m}^{-2}\right)$ \\
\hline 0.058 & $0.676 \times 10^{-6}$ \\
0.107 & $0.943 \times 10^{-6}$ \\
0.207 & $1.32 \times 10^{-6}$ \\
0.390 & $1.79 \times 10^{-6}$ \\
0.746 & $2.43 \times 10^{-6}$ \\
1.04 & $3.14 \times 10^{-6}$ \\
1.97 & $4.01 \times 10^{-6}$
\end{tabular}

Table 2. Experimentally measured values of surface concentration $\hat{\Gamma}$ versus bulk concentration $\hat{C}_{b}$ for the surfactants $\mathrm{C}_{14} \mathrm{TAB}$ and $\mathrm{C}_{16} \mathrm{TAB}$.

thus outweighed by the inconvenience of choosing between these possible limits as $S$ and $K$ are varied. We therefore treat $\beta$ as an $O(1)$, although numerically small, constant.

\section{Comparison with experiments}

We now compare the predictions of our mathematical model with experimental data from the overflowing cylinder experiment. We consider two sets of experimental data, the first using solutions of $\mathrm{C}_{14} \mathrm{TAB}$ with salt and the second using $\mathrm{C}_{16} \mathrm{TAB}$ with salt. The salt is intended to screen out any electrical effects and thus render the surfactant effectively non-ionic. Approximate values of the physical parameters for these two surfactants are summarised in table 1.

For each of these two surfactants, the bulk concentration $\hat{C}_{b}$ fed into the OFC is varied, 


\begin{tabular}{cccccccc}
\cline { 5 - 7 } & & & & $\hat{\Gamma}\left(10^{-6} \mathrm{~mol} \mathrm{~m}^{-2}\right)$ \\
\hline \hline$\hat{C}_{b}\left(\mathrm{~mol} \mathrm{~m}^{-3}\right)$ & $\theta\left(\mathrm{s}^{-1}\right)$ & $C_{b}$ & $S$ & Eqm & $k_{-1}=5 \mathrm{~s}^{-1}$ & $k_{-1}=2 \mathrm{~s}^{-1}$ \\
\hline 0.05 & 1.13 & 0.25 & 0.902 & 0.779 & 0.779 & 0.779 \\
0.1 & 1.61 & 0.5 & 1.08 & 1.30 & 1.30 & 1.30 \\
0.2 & 3.11 & 1.0 & 1.50 & 1.87 & 1.87 & 1.87 \\
0.285 & 4.48 & 1.43 & 1.80 & 2.09 & 2.07 & 2.03 \\
0.389 & 5.06 & 1.95 & 1.91 & 2.46 & 2.42 & 2.33 \\
0.6 & 5.32 & 3.00 & 1.96 & 3.15 & 3.07 & 2.92 \\
0.92 & 5.15 & 4.60 & 1.93 & 3.48 & 3.45 & 3.39 \\
1.2 & 3.71 & 6.0 & 1.63 & 3.53 & 3.51 & 3.50 \\
\hline \hline
\end{tabular}

Table 3. Experimentally measured values of bulk concentration $\hat{C}_{b}$ and surface expansion rate $\theta$ for $\mathrm{C}_{14} \mathrm{TAB}$, corresponding dimensionless parameters $C_{b}$ and $S$, and calculated values of $\hat{\Gamma}$ in the equilibrium limit, with $k_{-1}=5 \mathrm{~s}^{-1}$ and with $k_{-1}=2 \mathrm{~s}^{-1}$.

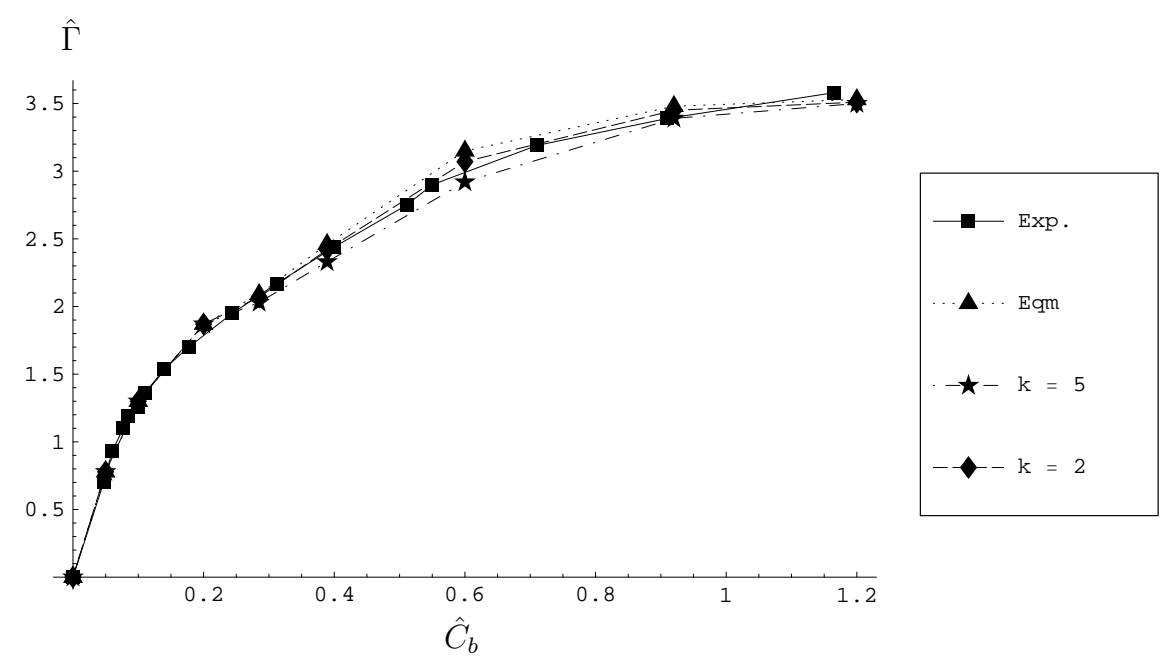

Figure 6. Dimensional surface concentration $\hat{\Gamma}$ versus dimensional bulk concentration $\hat{C}_{b}$ for $\mathrm{C}_{14} \mathrm{TAB}$; experimental values $(\boldsymbol{\square})$ and asymptotic predictions $(\boldsymbol{\Lambda})$ in the equilibrium limit $K \rightarrow$ $\infty,(\star)$ with $k_{-1}=5 \mathrm{~s}^{-1},(\diamond)$ with $k_{-1}=2 \mathrm{~s}^{-1}$.

and the corresponding surface concentration $\hat{\Gamma}$ is measured. The results are shown in table 2 .

In separate experiments (using different measurement tools), the surface expansion rate $\theta$ is measured as the bulk concentration $\hat{C}_{b}$ is varied. For each such experiment, we can thus calculate the remaining two dimensionless parameters $C_{b}$ and $S$. If the system is assumed to be in equilibrium, then we have enough information to determine $Z$ and $C_{0}$ from (4.5) and (4.9), and may then obtain the dimensional surface concentration $\hat{\Gamma}$ from (2.8). The results of this process for $\mathrm{C}_{14} \mathrm{TAB}$ are shown in table 3 . We also show the corresponding non-equilibrium predictions when $k_{-1}=5 \mathrm{~s}^{-1}$ and $k_{-1}=2 \mathrm{~s}^{-1}$.

Direct comparison between the values in tables 2 and 3 is difficult, since different bulk concentrations are used in either case. We therefore compare them graphically in figure 6 . 


\begin{tabular}{ccccccc}
\cline { 4 - 6 } & & & & $\hat{\Gamma}\left(10^{-6} \mathrm{~mol} \mathrm{~m}^{-2}\right)$ \\
\hline \hline$\hat{C}_{b}\left(\mathrm{~mol} \mathrm{~m}^{-3}\right)$ & $\theta\left(\mathrm{s}^{-1}\right)$ & $C_{b}$ & $S$ & Eqm & $k_{-1}=20 \mathrm{~s}^{-1}$ & $k_{-1}=10 \mathrm{~s}^{-1}$ \\
\hline 0.045 & 1.05 & 1.29 & 5.24 & 0.751 & 0.750 & 0.749 \\
0.1 & 1.28 & 2.86 & 5.78 & 1.23 & 1.22 & 1.21 \\
0.2 & 2.00 & 5.71 & 7.23 & 1.65 & 1.60 & 1.54 \\
0.46 & 3.52 & 13.1 & 9.59 & 2.42 & 2.13 & 1.92 \\
0.6 & 4.66 & 17.1 & 11.0 & 2.64 & 2.16 & 1.89 \\
0.92 & 5.03 & 26.3 & 11.5 & 3.66 & 2.76 & 2.34 \\
1.2 & 5.1 & 34.3 & 11.5 & 4.0 & 3.29 & 2.75 \\
2.5 & 4.29 & 71.4 & 10.6 & 4.0 & 3.96 & 3.93 \\
\hline \hline
\end{tabular}

Table 4. Experimentally measured values of bulk concentration $\hat{C}_{b}$ and surface expansion rate $\theta$ for $\mathrm{C}_{16} \mathrm{TAB}$, corresponding dimensionless parameters $C_{b}$ and $S$, and calculated values of $\hat{\Gamma}$ in the equilibrium limit, with $k_{-1}=20 \mathrm{~s}^{-1}$ and with $k_{-1}=10 \mathrm{~s}^{-1}$.

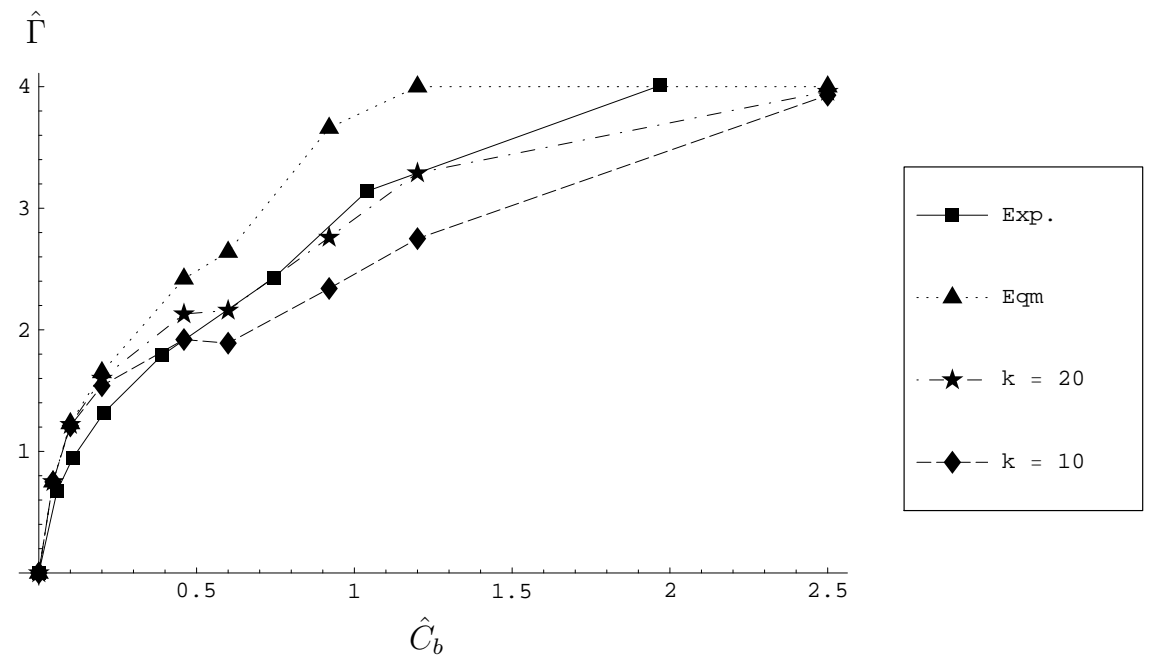

FiguRE 7. Dimensional surface concentration $\hat{\Gamma}$ versus dimensional bulk concentration $\hat{C}_{b}$ for $\mathrm{C}_{16} \mathrm{TAB}$; experimental values $(\boldsymbol{\square})$ and asymptotic predictions $(\boldsymbol{\Lambda})$ in the equilibrium limit $k \rightarrow$ $\infty,(\star)$ with $k_{-1}=20 \mathrm{~s}^{-1},(\diamond)$ with $k_{-1}=10 \mathrm{~s}^{-1}$.

The agreement between the equilibrium values and the experimental data is excellent, which both validates our simple model for micellar breakdown and indicates that the limit $K \rightarrow \infty$ gives a good approximation for this surfactant. In other words, the micellar and monomer phases were everywhere close to equilibrium in this set of experiments. In fact the experimental surface concentrations are systematically lower than the theoretical equilibrium values, and a slightly better fit is obtained with the $k_{-1}=5 \mathrm{~s}^{-1}$ data. We cannot necessarily infer the approximate value of $k_{-1}$ from this observation, since the slight departure from the equilibrium curve might also be caused by inaccuracies in the values of the other parameters such as $C_{\mathrm{cmc}}, \Gamma_{\mathrm{sat}}$ and $D$.

Next we perform the same procedure for $\mathrm{C}_{16} \mathrm{TAB}$. Again, with given experimental values of $\hat{C}_{b}$ and $\theta$, we can calculate $C_{b}, S$ and hence $\hat{\Gamma}$. The values obtained in the equi- 
librium limit $K \rightarrow \infty$ are listed in the fifth column of table 4 and compared graphically with measured values of $\hat{\Gamma}$ in figure 7 . Here, the predicted equilibrium surface concentration is significantly higher than the experimental values. We infer that the transport of surfactant to the surface is limited in this case by micellar breakdown. We also tabulate non-equilibrium predictions for $\hat{\Gamma}$ in table 4 , using values of $k_{-1}=20 \mathrm{~s}^{-1}$ and $k_{-1}=10 \mathrm{~s}^{-1}$.

These are also visualised in figure 7 , where we can see that reducing the rate constant $k_{-1}$ has the desired effect of reducing $\hat{\Gamma}$. In particular, $k_{-1}=20 \mathrm{~s}^{-1}$ gives rather good agreement with experiment. This suggests that the experiments using $\mathrm{C}_{16} \mathrm{TAB}$ were in a nonequilibrium regime and allows us to estimate the relevant rate constant $k_{-1} \approx 20 \mathrm{~s}^{-1}$.

\section{Discussion}

We have presented a model describing the distribution of monomer and micelles in a surfactant solution, and applied it to a stagnation point flow. As well as being particularly relevant to the so-called overflowing cylinder experiment, this linear straining flow also represents the canonical local behaviour near an expanding free surface. We first analysed a particularly simple limit in which the model equations may be integrated exactly. We then used the insight gained to construct leading-order asymptotic solutions of the full equations. These approximate solutions were compared both with numerical solutions and with experimental results.

Our asymptotic analysis reveals two limiting regimes: one in which the monomer solution is significantly below the CMC and one in which it is approximately at the CMC. The solution of the problem thus hinges on locating the free boundary separating regions where the two different regimes prevail. The analysis is particularly straightforward if the micelle and monomer phases are assumed everywhere to be in equilibrium, which amounts to assuming that the timescale for micelle breakdown is much shorter than any other timescale in the problem. In this limit, micelles only exist where the monomer solution is at the $\mathrm{CMC}$, and the free boundary satisfies a single transcendental equation. If the timescale for micelle breakdown is finite, the analysis is slightly more complicated, and involves matching across a thin boundary layer in which the transition from a micellar to a sub-micellar solution occurs. Nevertheless, the free boundary still satisfies a (rather complicated) transcendental equation.

Because we only consider steady states, our general model is a boundary-value problem for a system of ordinary differential equations. As an alternative to asymptotic analysis, these may also be directly solved numerically; we illustrated such solutions in section 4 . Our asymptotic reduction offers many advantages, though. By assuming that $n$ and $K$ are large, we remove any explicit dependence on these parameters from the problem. Moreover, the governing equations (2.20) and (2.21), containing terms like $C^{n}$ where $n \approx 100$, are numerically very stiff as they stand. If our theory were applied to more general flows, for which analytical solutions were unavailable, the numerical difficulty would still be greatly reduced by taking the limit $n \rightarrow \infty$.

We have chosen to consider only micelles of a given fixed size, thus lumping all intermediate aggregates into our effective reaction rates. Others, for example [13] and [21], have derived generalised models allowing micelles of many sizes to coexist. Due to the 
added complexity, though, these authors only consider concentrations that are close to their equilibrium values, while we are able to study systems that are far from equilibrium. Nevertheless, it would be interesting to discover whether the inclusion of intermediate reactions would affect our conclusions significantly, and we plan to pursue this question subsequently.

Our model is similar to that described in [10] for surfactant solution in a dilational flow. However, our method of solution is completely different. They invoke imaginary layers and prescribe the forms for monomer and micelle concentrations in each layer. We systematically solve the model using asymptotic and numerical techniques.

In the experiments, salt is used to help screen the electrical double-layer that forms when ionic surfactant is adsorbed at an interface. In practical applications, though, such ionic effects often play a crucial role in the behaviour of surfactant solutions, and their influence on the OFC model for sub-CMC solutions has been considered by [5]. To complete the picture, we plan to derive a general model incorporating ionic and micellar effects.

\section{Acknowledgements}

We are grateful for many useful discussions with Prof R. C. Darton, Dr C. D. Bain and Dr D. Valkovska, and to R. Campbell, M. Sekine and G. Shearman for the use of their overflowing cylinder data. This work was supported by EPSRC under grant GR/R52190/01.

\section{References}

[1] Adamson, A. W. 1982 Physical Chemistry of Surfaces. Wiley.

[2] Atkins, P. W. 1998 Physical Chemistry. Oxford.

[3] Bain, C. D., Manning-Benson, S. \& Darton, R. C. 2000 Rates of mass transfer and adsorption of hexadecyltrimethylammonium bromide at an expanding air-water interface. J. Colloid Interface Sci. 229, 247-256.

[4] Billingham, J. 2000 On modelling the formation of micelles in the presence of a slow influx of monomer. Q. J. Mech. App. Math. 53:(2), 285-297.

[5] Bosson, J. M., Breward, C. J. W. B. and Howell, P. D. 2003 Ionic effects in the overflowing cylinder experiment. Preprint.

[6] Breward, C. J. W., Darton, R. C., Howell, P. D. and Ockendon, J. R. 2001 The effect of surfactants on expanding free surfaces. Chem. Eng. Sci. 56, 2867-2878.

[7] Breward, C. J. W. and Howell, P. D. 2002 The drainage of a foam lamella. J. Fluid Mech. 458, 379-406.

[8] Chang, C-.H., and Franses, E. I. 1995 Adsorption dynamics of surfactants at the air-water interface: a critical review of mathematical models and mechanisms. Coll. Surf. A: Physicochem. Eng. Aspects 100, 1-45.

[9] Coveney, P. V., \& Wattis, J. A. D. 1996 Analysis of a generalised Becker-Doring model of self-reproducing micelles. Proc. Roy. Soc. A 452, 2079-2102.

[10] Danov, K. D., Vlahovska, P. M., Horozov, T., Dushkin, C. D., Kralchevsky, P. A., Mehreteab, A. and Broze, G. 1996 Adsorption from micellar surfactant solutions: Nonlinear theory and experiment. J. Coll. Int. Sci. 183 223-235.

[11] Denbigh, K. 1981 The Principles of Chemical Equilibrium, 4th Edition. Cambridge.

[12] Diamant, H., Ariel, G. and Andelman, D. 2001 Kinetics of surfactant adsorption: the free energy approach. Coll. Surf. A: Physicochem. Eng. Aspects 183, 259-276. 
[13] Dushkin, C. D. 1998 Model of the quasi-monodisperse micelles with application to the kinetics of micellization, adsorption and diffusion in surfactant solutions and thin liquid films. Coll. Surf. A: Physicochem. Eng. Aspects 143, 283-299.

[14] Gradshteyn, I. S. and Ryzhik, I. M. 1994 Tables of Integrals, Series, and Products, Fifth Edition. Academic.

[15] Howell, P. D. and Breward, C. J. W. 2003 Mathematical modelling of the overflowing cylinder experiment. J. Fluid Mech. 474, 275-298.

[16] Lee, Y.-C., Lin, S.-Y. and Lie, H.-S. 2001 Role of equation of state on studying surfactant adsorption kinetics. Langmuir 17, 6196-6202.

[17] Levich, V. G. 1961 Physicochemical Hydrodynamics. Prentice-Hall.

[18] Lin, S.-Y., Lee, Y.-C., Shao, M.-J., and Hsu C.-T. 2001 A study on surfactant adsorption kinetics: the role of the data of equation of state $\gamma(\Gamma)$ for $\mathrm{C}_{14} \mathrm{E}_{8}$. J. Coll. Int. Sci. 244, $372-376$.

[19] Manning-Bension, S., Bain, C. D. \& Darton, R. C. 1997 measurement of dynamic interfacial properties in an overflowing cylinder by ellipsometry. J. Coll. Int. Sci. 89, 109-116.

[20] Manning-Bension, S., Bain, C. D., Darton, R. C., Sharpe, D., Eastoe, J. \& Reynolds, P. 1997 Invasive and non-invasive measurements of dynamic surface tensions. Langmuir 13, $5808-5810$.

[21] Noskov, B. A. 2002 Kinetics of adsorption from micellar solutions. Adv. Coll. Int. Sci. 95, 237-293.

[22] Schwartz, L. W. and Princen, H. M. 1987 A theory of extensional viscosity for flowing foams and concentrated emulsions. J. Coll. Int. Sci. 118, 201-211.

[23] Shaw, D. W. 1992 Colloid and surface chemistry $4^{\text {th }}$ Edition. Butterworth-Heinemann LTD.

[24] Valkovska, D. S., Shearman, G. C., Bain, C. D., Darton, R. C. and Eastoe, J.2003 Adsorption of ionic surfactants at an expanding air-water interface. Submitted to Langmuir. 